\title{
Retroviruses: delivery vehicle to the liver
}

\author{
Mariann Grossman and James M. Wilson
}

\author{
University of Michigan, Ann Arbor, Michigan, USA
}

\begin{abstract}
Liver-directed gene therapy holds great promise for the treatment of inherited metabolic disease. Two strategies have emerged. Ex vivo gene therapy involves the transplantation of autologous hepatocytes transduced with recombinant retroviruses while in culture. The feasibility of this approach has been demonstrated in several animal models, and a human trial has been initiated. An alternative strategy uses recombinant viruses to deliver the transgene directly to hepatocytes in vivo.
\end{abstract}

Current Opinion in Genetics and Development 1993, 3:110-114

\section{Introduction}

Inborn errors of metabolism comprise a diverse group of rare, disabling disorders that collectively contribute to substantial morbidity in our society. A large number of these disorders are caused by defects in enzymes that are primarily expressed in the liver and which are involved in intermediary metabolism [1]. Three examples include: ornithine transcarbamylase deficiency (OTCD), which is characterized by an accumulation of ammonia leading to episodes of coma; familial hypercholesterolemia (FH), which is caused by a defect in low density lipoprotein (LDL) receptor and is characterized by an extreme elevation in LDL cholesterol leading to the development of premature coronary heart disease; and phenylketonuria (PKU), which is caused by a defect in phenylalanine hydroxylase and is characterized by mental retardation if not properly managed. Several strategies have been developed for treating inborn errors of metabolism: first, counseling and/or patient education; second, metabolite intervention either by pharmacologic replacement of the product, dietary restriction, or by utilizing an altemative enzymatic pathway; third, activation or replacement of the dysfunctional protein; and finally, organ transplantation to replace the defective liver function. Despite extensive research in the development of these and other innovative therapies most of these diseases are essentially refractory to anything except palliative therapy.

Several metabolic diseases have been treated by orthotopic liver transplantation. In many situations, this was performed in an attempt to treat secondary consequences of the primary molecular defects that lead to generalized destruction of liver function. However, orthotopic liver transplantation has also been indicated in lethal metabolic diseases as a means of correcting the primary genetic defect in liver function. In this application, the liver is structurally normal and the transplant is per- formed to replace a single defective metabolic derangement. Orthotopic liver transplantation for metabolic disease has been performed in a limited number of patients with the following diseases: $\mathrm{FH}, \boldsymbol{\alpha} \cdot 1$-antitrypsin deficiency, Crigler-Najjar syndromel; and a few glycogen-storage diseases [2]. In pediatric cases, the five-year survival rate following liver transplantation for metabolic diseases is approximately $83 \%$. Mortality can occur in the perioperative period, while chronic immunosuppressive therapy and the ever-present threat of rejection contributes to ongoing morbidity. Because of these unavoidable complications, it is difficult to justify a liver transplant in all but the most severe conditions. A more attractive approach would be to establish methods for selectively reconstituting the expression of the functional gene in the liver by somatic gene therapy.

An alternative approach to organ transplantation is $e x$ vivo gene therapy. The process of ex vivo gene transfer entails harvesting the appropriate tissue, isolating the target cell, growing the cells in vitro, introducing the gene of interest into the cells with recombinant retroviruses, and placing the genetically modified cells back into the patient from which they were derived. An important early paradigm of ex vivo gene therapy has been strategies based on the transplantation of genetically modified hepatocytes.

The first step of liver-directed ex vivo gene therapy is the isolation of viable hepatocytes from biopsied tissue. Important limitations in the process of ex vivo gene therapy directed to the liver is the inability to expand isolated hepatocytes in culture. It is possible to establish primary cultures but impossible to passage these cells. Thus, to obtain therapeutic quantities of hepatocytes for genetic modification, it is necessary to resect significant portions of tissue and genetically modify the cells soon after isolation.

$$
\begin{aligned}
& \text { Abbreviations } \\
& \text { FH—familial hypercholesterolemia; LDL-low density lipoprotein; LTR-long terminal repeat; } \\
& \text { OTCD—ornithine transcarbamylase deficiency; PKU—phenylketonuria; WHHL-Watanabe heritable hyperlipidemic. }
\end{aligned}
$$




\section{Hepatocyte isolation}

Hepatocytes have been isolated from a variety of species including mouse, rat, rabbit, dog, baboon and human tissue $[3,4,5 \cdot \bullet, 6,7 \bullet, 8 \bullet, 9 \bullet \bullet, 10-16]$. The general approach is to perfuse liver tissue with a neutral protease to release individual cells. Fractions of cells enriched for hepatocytes are obtained by differential sedimentation. The enriched suspension of hepatocytes are plated in culture and cultivated in a hormonally defined media. (See the references $[3,4,5 \bullet \cdot, 6,7 \bullet, 8 \cdot, 9 \bullet \bullet, 10-17]$ both for examples of the numerous species from which hepatocytes have been isolated, and for published experiences of the isolation and in vitro cultivation of hepatocytes.) Despite strategies for enrichment, hepatocyte cultures are never pure; one frequently recovers cultures that contain greater than $95 \%$ hepatocytes but there are always a number of contaminating cells within these cultures (e.g., endothelial cells, Kupffer cells, ito cells or biliary epithelial cells). The quality of hepatocyte preparations, which is most frequently measured in terms of total yield and viability, varies considerably.

Extensive literature exists on the primary cultivation of hepatocytes in vitro. A variety of conditions have been established for maintaining hepatocytes in culture. Despite tremendous efforts it has been impossible to establish conditions necessary to passage and expand adult hepatocytes. In addition, it is difficult to maintain the differentiated state of the cells for prolonged periods in culture. In a typical experiment, hepatocytes can be maintained in culture for approximately 14 days, during which time the cells undergo several rounds of cell division.

\section{Gene transfer into hepatocytes using retroviruses}

Several approaches have been developed for transferring genes into cultured hepatocytes. The traditional approaches of transfection such as calcium phosphate transfection, electroporation, and lipofection have been used; however, expression of the recombinant gene has been extremely low and transient [18-21]. Furthermore, these approaches are associated with substantial in vitro toxicity. The most successful strategy has been to use recombinant retroviruses.

Retroviruses are enveloped viruses containing a singlestranded RNA genome [22]. A typical retrovirus expresses three essential structural genes: gag, encoding a collection of proteins involved in virus assembly; pol, encoding a multifunctional protein containing several activities including reverse transcriptase and an integrase; and $e n v$, encoding a glycoprotein that spans the envelope and contains a domain on the surface of the virion that is recognized by a cell surface receptor. Retroviruses bud from the surface of infected cells in a non-lytic manner. Infection begins with endocytosis of the virus and delivery of the RNA genome into the cytoplasm through a fusion event between the virion envelope and the membrane of the endosome that is mediated by the fusigenic envelope protein. The RNA is converted to a double-stranded DNA intermediate through the sequential activity of reverse transcriptase, RNase $\mathrm{H}$, and DNA polymerase. The DNA is very efficiently integrated into the genome of the recipient cell in a relatively random manner. The integrated form of the virus, called the provirus, contains repeated sequences, termed long terminal repeats (LTRs), at both ends. The provirus contains a single transcriptional unit that is initiated at the $5^{\prime}$ LTR. This full-length transcript, called the genomic RNA, has two functions: first, it contains a sequence, called $\psi$, that enables it to be packaged into virions; and second, it can be translated, yielding the Gag and Pol proteins. The genomic transcript undergoes processing to form the subgenomic transcript that can be translated into the Env protein.

Several strategies have been suggested for using retroviruses to transduce recombinant genes. One approach is to splice a recombinant gene into a complete retroviral genome. The resulting recombinant virus will transduce the recombinant gene; however, it will remain infectious, with recipient cells producing recombinant viruses. Several investigators have suggested strategies for generating recombinant retroviruses that are replication defective. The general approach is to generate a packaging cell line that can provide in trans all the proteins necessary to form virions. This is accomplished by transfecting into a mouse fibroblast cell line a retroviral genome from which the $\psi$ sequences have been deleted. The genomic transcript can express gag, pol and env; however, it.is not packaged into virions. A cell line that produces recombinant virus is generated by transfecting into the packaging cell line a retroviral vector in which the gag, pol and env sequences have been replaced with a recombinant gene. The genomic transcript from the retroviral vector contains $\psi$ sequences, and thus it is incorporated into virions of the packaging cell line. Resulting viruses transduce the recombinant gene and are replication-defective because they do not transduce gag, pol and env. Packaging cell lines are available with different tropisms based on the envelope proteins that they express [23-25]. Ecotropic cell lines express viruses whose tropism is restricted to rodents, while virus from amphotropic cell lines have extremely wide host ranges and are capable of infecting most mammalian cells, including humans.

A critical step in the development of ex vivo gene therapy for the liver is to stably transfect a large proportion of cultured hepatocytes before transplantation of the corrected autologous cells. The limitations of primary hepatocyte cultures described above provide difficult challenges in achieving this. First, hepatocytes cannot be passaged, and thus selection and expansion of the transduced cells is not possible. Furthermore, the cells undergo few cell divisions and remain differentiated and viable for only a short period of time. Therefore, gene transfer must occur during a short window after plating and it must be extremely efficient. The most promising approach has been with recombinant retroviruses. Wolff et al [16] were the first to demonstrate transduction of hepatocytes using a neo expressing retrovirus and adult rat hepatocytes. Success- 
ful transduction was further demonstrated using lac $Z$ expressing retroviruses in rat hepatocytes and neo-expressing retroviruses with newborn mouse hepatocytes $[13,15]$. Under optimal circumstances it is possible to achieve transduction efficiencies that approach 30-50\% with a single exposure to virus. It is critical that the cells are plated at subconfluent densities so that they can undergo at least one round of cell division. The most important factor in achieving efficient gene transfer is the time after plating that the cells are exposed to virus. We and others have identified a window between $48-72 \mathrm{~h}$ after plating during which the cells are most susceptible to infection. The mechanisms for this are unclear; however it may relate, in part, to the fact that the cells are most actively cycling during this time. Retrovirus-mediated gene transfer has now been demonstrated in cells from a variety of species including adult and newbom mouse, rat, adult and newborn rabbit, dog, baboon, and human $[6,7 \bullet, 8 \bullet, 11,14-16,26]$. In addition, a variety of potentially therapeutic recombinant genes have been transduced and expressed in hepatocytes. These include genes expressing the LDL receptor, omithine transcarbamylase, phenylalanine hydroxylase, factor IX, and UDP glucuronosyltransferase (JM Wilson and F Askari, unpublished data) $[4,5 \bullet \bullet, 6,11,14,26]$.

\section{Hepatocyte transplantation}

Strategies for harvesting and transplanting the genetically modified hepatocytes are currently being developed. For purposes of ex vivo gene therapy it is necessary to design approaches that enable the ex vivo manipulated cells to be efficiently harvested and transplanted, and allow the cells to stably engraft and continue express. ing the recombinant gene. Initial attempts to transplant hepatocytes used unmarked syngeneic cells that were introduced into sites such as the dorsal fat pad or spleen $[27,28]$. Long-term engraftment of these cells was documented by demonstrating persistence of cells with hepatocyte morphology in the ectopic sites. These strategies are not suitable for therapeutic application because the number of cells that can be engrafted are extremely limited. An approach of more relevance to gene therapy is to demonstrate correction of hepatocyte dysfunction by virtue of cell transplantation. This has been attempted in several animal models. Transplantation of syngeneic hepatocytes into the portal circulation of rats following 90\% partial hepatectomy resulted in improved survival, indicating short-term function of the cells until the remnant liver regenerated [29]. Rats genetically deficient in albumin achieved transient levels of detectable serum albumin when transplanted with allogeneic hepatocytes into the portal vein or peritoneal cavity [30]. The production of albumin was prolonged when the recipient was immunosuppressed or the donor cells were purged of macrophages. Stable correction of defective bilirubin metabolism was achieved in the Gunn rat transplanted with congenic hepatocytes [31]. Similar principles have been applied with transgenic mice [32-34]. Hepatocytes from animals carrying a liver-specific transgene have been transplanted into congenic recipients. Engraftment of the donor hepatocytes, detected by virtue of transgene expression, has been demonstrated for at least one year. The first trials of hepatocyte transplantation in humans were conducted in Japan. Allogeneic hepatocytes were transplanted into the spleens of patients suffering from fulminant liver failure [35].

Lessons learned from models of hepatocyte transplanta. tion have now been applied to models of ex vivo gene therapy. The major difference is that the cells used in the transplantation studies described above were freshly isolated, whereas those used in ex vivo gene therapy must be cultured for at least three days - during their transduction with retroviruses - before they are harvested for transplantation. Anderson et al. [3] achieved short-term engraftment of syngeneic hepatocytes that were transduced with a neo virus and transplanted onto a sponge implanted into the peritoneal cavity of rats. We have used the human disease FH as a model for developing ex vivo gene therapies for the liver. The availability of an animal model of this disease, termed the Watanabe heritable hyperlipidemic (WHHL) rabbit, has been extremely useful in developing gene-therapy strategies. Transplantation of allogeneic hepatocytes transduced with a retrovirus containing the human LDL receptor gene into WHHL rabbits led to temporary amelioration of hypercholesterolemia [14]. Loss of in vivo efficacy of the therapy was the result of destruction of the transplanted cells, presumably because of an immune response directed at the allogeneic cells or the human LDL-receptor protein. These problems were overcome in a second series of experiments in which autologous hepatocytes transduced with retroviruses containing a normal rabbit LDLreceptor gene were transplanted into the portal circulation of WHHL rabbits [5••]. Recipient animals realized a prolonged improvement in serum cholesterol; analysis of liver tissue demonstrated high levels of recombinant-derived LDL-receptor RNA for the duration of the experiment ( 6.5 months). The practicality of this approach has been demonstrated in larger animals, including dogs and baboons $[8 \cdot, 9 \cdot \bullet]$.

\section{In vivo gene transfer using retroviruses}

Recent studies have demonstrated the feasibility of using recombinant retroviruses to deliver genes to hepatocytes in vivo [36]. The major problem is that hepatocytes are normally quiescent and efficient retroviral infection requires a population of target cells that are dividing. Hatzglou et al. [37] injected recombinant viruses into the peritoneal cavity of developing rats at a time when the fetal liver is actively growing. Using a recombinant virus with a liver-specific promoter, they were able to demonstrate transduction of hepatocytes. Similar results have been achieved in adult rats when the virus is infused into animals following injury to the liver, such as partial hepatectomy, to stimulate cell division in the target hepatocytes. Infusion of retrovirus into the portal circulation 
of rats following partial hepatectomy resulted in stable transduction in a small population of hepatocytes based on PCR analyses [38]. The efficiency of hepatocyte transduction is increased 1-5\% when the liver is temporarily devascularized and infused with virus $24 \mathrm{~h}$ after partial hepatectomy [39].

\section{Conclusions}

Gene transfer directed to hepatocytes has emerged as an important early paradigm of gene-replacement therapies. Recombinant retroviruses have been used in both ex vivo and in vivo approaches to liver-directed gene therapies. The rational and feasibility of ex vivo gene therapy for liver metabolic disease has been established. While this form of therapy will probably have important applications, it is complicated and impractical for broad use. In addition, the efficiency is limited by the number of autologous cells that can be harvested and re-infused into the portal circulation. Importantly, the duration of therapeutic effect achieved using this approach is unknown because the gene is not inserted into a stem cell. In vivo gene therapy provides the potential of more efficient gene transfer and the feasibility of repeated treatments. However, approaches for stimulating hepatocyte division that are more practical and less morbid than partial hepatectomy will need to be developed.

\section{References and recommended reading}

Papers of particular interest, published within the annual period of review, have been highlighted as:

- of special interest

-. of outstanding interest

1. SCRIVE CR, Beaudet AL, SLY WS, VAlle D: The Metabolic Basis of Imberiled Diseases, vol 2, 6th edn. New York: McGraw-Hill; 1989.

2. Esquivel CO, Marino IR, floravanti V, Van Thiel DH: Liver Transplantation for Metabolic Disease of the Liver. Liver Transplantation. Gastroenterology Clinics of North America $1988,17: 167-174$

3. ANDERSON KD, THOMPSON JA, DIPIETRO JM, MONTGOMERY KT REID LM, ANDERSON WF: Gene Expression in Implanted Rat Hepatocytes Following Retroviral-mediated Gene Transfer. Somat Cell Mol Genet 1989, 15:215-227.

4. ARMentano D, ThOMPSON AR, DARUINGTON G, WOO SL: Expression of Human IX in Rabbit Hepatocytes by Retrovirusmediated Gene Transfer: Potential for Gene Therapy of Hemophilia B. Proc Natl Acad Sci USA 1990, 87:6141-6145.

5. ChOWdURY JR, GROSSMAN M, GUPTA S, ChOWDURY NR, BAKER -. JR JR, WILSON JM: Long-term Improvement of Hypercholesterolemia After Ex Vivo Gene Therapy in LDLR-deficient Rabhits. Science 1991, 254:1802-1805.

This reference details a major advance in ex vivo gene therapy in animal models. An animal model for FH, the WHHL rabbit, was used to develop an approach for liver-directed gene therapy based on the transplantation of autologous hepatocytes that were genetically corrected ex vivo with recombinant retroviruses containing the LDL receptor. The animals demonstrated a 30-50\% decrease in total serum cholesterol that persisted for the duration of the experiment (122 days). Recombinant-de- rived LDL-receptor RNA was harvested from tissues with no diminution for up to 6.5 months after transplantation.

6. GRompe M, JoNES SN, LOULSEgED H, CASKEY CT: Retroviralmediated Gene $T$ Transfer of Human Ornithine Transcarbamlyase into Primary Hepatocytes of spf and spf-ash Mice. Hum Gene Ther 1992, 3:35-44.

7. GROSSMAN M, RAPER SE, WILSON J: Towards Liver-directed - Gene Therapy: Retrovirus-mediated Transfer into Human Hepatocytes. Somat Cell Mol Genet 1991, 17:601-607.

This paper is the first to describe the techniques for isolating and genetically modifying human hepatocytes and represents a critical step towards the development of liver-directed gene therapies in humans. Hepatocytes were isolated from four different donors that varied in age, sex and time post-perfusion. They were planted in primary culture and exposed to recombinant retroviruses expressing either the lac $Z$ reporter gene or the CDNA for rabbit LDL receptor.

8. GROSSMAN M, RAPER SE, WILSON JM: Transplantation of - Genetically-modified Autologous Hepatocytes in Non-human Primates. Hum Gene Ther 1992, 3:501-510.

This paper details experiments in non-human primates that document both the feasibility and safety of autologous hepatocyte transplantation. Three baboons underwent a partial hepatectomy and their hepatocytes were isolated, cultured and transduced with a retrovirus containing the human LDL-receptor gene. The hepatocytes were harvested and infused into an indwelling catheter. The baboons are being maintained and clinically evaluated for an indefinite period of time.

9. Kay MA, Bajey P, Rothenderg S, leland F, Fleming L, Ponder .. KP, LIU T, FINEGOLD M, DARIINGTON G, POKORNY W, ET AL. Expression of Human $\alpha 1$-antitrypsin in Dogs After Autologous Transplantation of Retroviral Transduced Hepatocytes. Proc Natl Acad Sci USA 1992, 89:89-93.

The second major advancement of ex vivo gene therapy in an animal model. The authors demonstrate the autologous transplantation of canine hepatocytes that had been transduced with a retroviral vector containing the human $\alpha$-1-antitrypsin CDNA under transcriptional control of the cytomegalovirus promoter. They were able to detect significant human $\alpha$-1-antitrypsin in the serum for one month with serum levels eventually decreasing because of inactivation of the $\mathrm{cy}$. tomegalovirus promoter.

10. KOCH KS, BROWNIEE GG, GOSS SJ, MARTINEZ-CONDE A, LEFFERT HL: Retroviral Vector Infection and Transplantation in Rats of Primary Fetal Rat Hepatocytes. J Cell Sci 1991, 99:121-130.

11. LEDLEY FD, DARIINGTON GJ, HAHN T, WOO SL: Retroviral Gene Transfer into Primary Hepatocytes: Implications for Genetic Therapy of Liver-specific Functions. Proc Natl Acad Sci USA 1987, 84:5335-5339.

12. Mryanohara A, Elam RL, WITZUM JL, FrIedmann T: Long-term Transgene Expression from Genetically Modified Hepatocytes Grafted to the Rat Liver. $N$ Biol 1992, 4:261-267.

13. Peng $H$, armentano $D$, Mackenzie.Graham L, SHEN RF, DARIINGTON G, LEDIEY FJ, WOO SL: Retroviral-mediated Gene Transfer and Expression of Human Phenylalanine Hydroxylase in Primary Mouse Hepatocytes. Proc Natl Acad Sci USA 1988, 85:8146-8150.

14. WILSON JM, ChOWDURY NR, GROSSMAN M, WAUSMAN R, EPSTEIN A MULUGAN RC, CHOWDURY JR: Temporary Amelioration of Hyperlipidemia in Low Density Lipoprotein Receptordeficient Rabbits Transplanted with Genetically Modified Hepatocytes. Proc Natl Acad Sci USA 1990, 87:8437-8441.

15. WIISON JM, JefFerson DM, ChOWDURY JR, NOVIKoFF PM, JOHNSTON DE, MULIGAN RC: Retrovirus-mediated Transduction of Adult Hepatocytes. Proc Natl Acad Sci USA 1988, 85:3014-3018.

16. WOLFF JA, YeE JK, SKeUY K, MOORES J, Respess J, Friedmann T LEFFERT HL: Expression of Retrovirally Transduced Genes in Primary Cultures of Adult Rat Hepatocytes. Proc Natl Acad Sci USA 1987, 84:3344-3348.

17. Friedmann T, XU L, WOlFF J, YeE JK, MIYanohara A: Retrovirus Vector-mediated Gene Transfer into Hepatocytes. Mol Biol Med 1989, 6:117-125. 
18. Gnfot F, DEchuX JF, COGNET M, BERBAR T, LEVRAT F, KAHN A, WEBER A: Transfection of Hepatic Genes into Adult Rat Hepatocytes in Primary Culture and their Tissue-specific Expression. Eur J Biocbem 1989, 180:289-294.

19. PASCO DS, FAGAN JB: Efficient DNA-mediated Gene Transfer into Primary Cultures of Adult Rat Hepatocytes. DNA 1989, 8:535-541.

20. RIPPE RA, BRENNER DA, LEFFERT HL: DNA-mediated Gene Transfer into Adult Rat Hepatocytes in Primary Culture. Mól Cell Biol.1990, 10:689-695.

21. TUR-Kaspa R, TEICher L, LEVINE BJ, SKOUlTChI AI, ShafritZ DA: Use of Elęctroporation to Introduce Biologically Active Foreign Genes into Primary Rat Hepatocytes. Mol Cell Biol 1986, 6:716-718.

22. VAN BEVEREN F, COFFIN J, HughES SH: RNA Tumor Viruses, 2nd edn. New York: Cold Spring Harbor Laboratory Press; 1985.

23. DANOS O, MUUJGAN RC: Safe and Efficient Generation of Recombinant Retroviruses with Amphotropic and Ecotropic Host Ranges. Proc Natl Acad Sci USA 1988, 85:6460-6464.

24. Markowitz D, GOFF S, BANK A: A Safe Packaging line for Gene Transfer: Separating Viral Genes on Two Different Plasmids. J Virol 1988, 62:1120-1124.

25. MnJER AD, BUTTIMORE C: Redesign of Retrovirus Packaging Cell Lines to Avoid Recombination Leading to Helper Virus Production. Mol Cell Biol 1986, 6:2895-2902.

26. WILSON JM, JOHNSTON DE, JEFFERSON DM, MUUJGAN RC: Correction of the Genetic Defect in Hepatocytes from the Watanabe Heritable Hyperlipidemic Rabbit. Proc Natl Acad Sci USA 1988, 85:4421-4425.

27. JIRTLE RL, BILES C, MiChalOPOULOS G: Morphologic and Histochemical Analysis of Hepatocytes Transplanted into Syngeneic Hosts. Am J Pathol 1980, 101:115-126.

28. Mrto M, Ebata H, Kusano M, Onishi T, Satro T, Sakamoto S: Morphology and Function of Isolated Hepatocytes Transplanted into Rat Spleen. Transplantation 1979, 28:499-505.

29. Gupta S, Johnstone R, Darby H, SeIden C, PRice Y, Hodgson H]: Transplanted Isolated Hepatocytes: Effect of Partial Hepatectomy on Proliferation of Long-term Syngeneic Implants in Rat Spleen. Patbology 1987, 19:28-30.

30. DEMETRIOU AA, WHITING JF, FELDMAN D, LEVENSON SM, CHOWDHURY NR, MOSCIONI AD, KRAM M, CHOWDHURY JR: Replacement of Liver Function in Rats by Transplanta- tion of Microcarrier-attached Hepatocytes. Science 1986 , 233:1190-1192.

31. Demetriou AA, WhIting J, LeVEnSON SM, ChOWdHuRY NR SCHECHNER R, MichaisKI S, FEIDMAN D, CHOWdHURY JR: New Method of Hepatocyte Transplantation and Extracorporeal Liver Support. Ann Surg 1986, 204:259-271.

32. Gupta S, ARAGona E, VEmuru RP, Bhargava KK, Burk RD, CHOWDURY JR: Permanent Engraftment and Function of Hepatocytes Delivered to the Liver: Implications for Gene Therapy and Liver Repopulation. Hepatology 1991 , 14:144-149.

33. Gupta S, Chowdury NR, Jagtiani R, Gustin K, ARagona E, SHAFrTZ DA, ChOWDURY JR, BURK RD: A Novel System for Transplantation of Isolated Hepatocytes Utilizing HBsAGproducing Transgenic Donor Cell. Transplantation 1990, $50: 472-475$.

34. PONDER KP, Gupta S, Leland F, DarLington G, Finegold $M$, Demayo J, Ledley FD, ChOWdury JR, WOO SL: Mouse Hepatocytes Migrate to Liver Parenchyma and Function Indefinitely after Intrasplenic Transplantation. Proc Natl Acad Sci USA 1991, 88:1217-1221.

35. MTto $M$, Kusano $M$, KaWaura $Y$ : Hepatocyte Transplantation in Man. Transplant Proc 1993, in press.

36. Clapp DW, DUMenCo LI, HATZglou M, GerSon SL: Fetal Liver Hematopoietic Stem Cells as a Target for in Utero Retroviral Gene Transfer. Blood 1991, 78:1132-1139.

37. Hatzglou M, lamers W, Bosch F, Wynshaw-Boris a, Clapp DW, HansoN RW: Hepatic Gene Transfer in Animals Using Retroviruses Containing the Promoter from the Gene for Phospoenolpyruvate Carboxykinase. J Biol Cbem 1990, 265:17285-17293.

38. Kaleko M, Garcia JV, Miller ad: Persistent Gene Expression after Retroviral Gene Transfer into Liver Cells in Vivo. Hum Gene Ther 1991, 2:27-32.

39. Ferry N, Duplessis O, houssin D, Danos O, Heard JM: Retroviral-mediated Gene Transfer into Hepatocytes in Vivo. Proc Natl Acad Sci USA 1991, 88:8377-8381.

M Grossman, JM Wilson, Departments of Internal Medicine and Biological Chemistry, and Howard Hughes Medical Institute, University of Michigan, Ann Arbor, Michigan 48104, USA. 